\title{
Many-to-Many Traffic Grooming in WDM Mesh Networks
}

\author{
Mohammad A. Saleh Ahmed E. Kamal \\ Department of Electrical and Computer Engineering, Iowa State University, Ames, IA 50011 \\ Email: \{msaleh,kamal $\} @$ iastate.edu
}

\begin{abstract}
In many-to-many communication, a session consists of group of users (we call them members) where each one of the members transmits its traffic to all other members in the group. This paper studies the problem of provisioning manyto-many sessions with sub-wavelength granularities in WDM mesh networks. Our objective is to minimize the number of transceivers required. We study the problem in networks with and without optical splitting capabilities. For networks without optical splitting capabilities, we use an optimal approach which is an extension of that introduced in [8] for the many-to-one traffic grooming problem. For networks with optical splitting capabilities, we introduce a novel hub-based approach where each session is routed through a many-to-one tree from the members to a central hub node, and then through a multicast tree from the hub node back to the members. At the hub node, network coding is performed by linearly combining the traffic units received from the members. These combinations are then groomed and delivered back to the members using light-tree(s). Numerical results from both approaches are presented and compared.
\end{abstract}

\section{INTRODUCTION}

In optical wavelength division multiplexing (WDM) networks, traffic grooming was introduced to reduce the huge gap between bandwidth requirements of user sessions and the bandwidth of a wavelength channel. For example, an MPEG compressed HDTV channel requires less than $20 \mathrm{Mbps}$ of bandwidth while the bandwidth of a wavelength channel may reach $10 \mathrm{Gbps}$. In addition to determining the virtual topology and the routing and wavelength assignment of each of the wavelength channels, the traffic grooming problem deals with the intelligent assignment of sub-wavelength traffic demands onto the existing wavelength channels. Most of the work in this area has focused on unicast traffic [1], [2], [3]. For a survey of advances in unicast traffic grooming, the reader is referred to [4].

In the recent years, more and more of the traffic in high performance networks is becoming of the multipoint type. This traffic type includes multicast, many-to-one and many-tomany. In many-to-many, a session consists of group of users (we call them members) where each one of these members transmits its traffic to all other members in the group. Ondemand video distribution and file distribution are examples of multicast applications, while resource discovery and data collection are examples of many-to-one applications. In the case of many-to-many where several users interact together, multimedia conferencing, distance learning, distributed simulations,

This research was supported in part by grant CNS-0626741 from the National Science Foundation. and collaborative processing are some of the applications. Since WDM networks provide the capacity to accommodate these high-bandwidth applications, finding efficient ways of provisioning these applications at the optical layer has become prominent.

Traffic grooming for multicast and many-to-one traffic types has been studied in the literature [5], [6], [8]. For an account of recent advances in multicast traffic grooming, the reader is referred to [7]. To the best of our knowledge, the problem of many-to-many traffic grooming in WDM networks has not been addressed before. In this problem, one is given a set of many-to-many sessions with arbitrary sub-wavelength traffic demands and the objective is to minimize the number of electronic ports which are used to add or drop a certain wavelength at a node (e.g., number of transceivers).

In order to effectively support multicast and many-to-many traffic types, nodes in a WDM network must be able to duplicate incoming traffic into multiple copies each going to a different output port. Two main node architectures were proposed in the literature to implement this functionality. In the first one, nodes can only duplicate an incoming optical signal by applying optical-electronic-optical $(O / E / O)$ conversion to the signal and duplication takes place in the electronic domain, we refer to networks with these nodes as non-splitting networks. In the second one, nodes are capable of splitting the incoming signal in the optical domain. Therefore, duplication can take place in the optical domain, we refer to networks with these nodes as splitting networks. In this paper, we study the many-to-many traffic grooming problem in both splitting and non-splitting networks.

The rest of the paper is organized as follows. In Section II, we propose the lightpath approach and the hub-based approach to solve the many-to-many traffic grooming problem in non-splitting and splitting WDM networks, respectively. In Section III, we formulate an MILP as an analytical model for the hub-based approach. Numerical results from both approaches are presented and compared in Section IV, while the paper is concluded in Section V.

\section{MANY-TO-MANY TRAFFIC GROOMING: NON-SPLITTING VERSUS SPLITTING NETWORKS}

\section{A. Many-to-Many Traffic Grooming in non-Splitting Networks}

In non-splitting networks, lightpaths will be the only optical communication channels available to provision many-to-many 
sessions. To optimally solve the many-to-many traffic grooming problem, we have generalized the many-to-one MILP in [8] to accommodate sessions of multiple sources and multiple destinations. Each source in the source set transmits its traffic to all destinations in the destination set. Accordingly, a manyto-many session will simply be represented as a session whose source set and destination set are the same and they correspond to the members of the many-to-many session. In this model, a many-to-many session can traverse multiple lightpaths from any member to any other member in the group, while the lightpath itself can traverse multiple fibers. The objective is to minimize the total number of transceivers. For the rest of the paper, we refer to this approach as the lightpath approach and refer to the generalized MILP as LP-MILP. We do not include the LP-MILP in this paper due to space limitations; however, it is available online [10] for interested readers.

\section{B. Many-to-Many Traffic Grooming in Splitting Networks}

In splitting networks, light-trees, in addition to lightpaths, can be used to provision many-to-many sessions. In this section, we introduce a novel hub-based approach to provision many-to-many sessions in splitting networks. In this approach, all of the members, in a session with $N$ members, send their traffic units to a central hub node which can be any node in the network including the members themselves. This hub node then linearly combines the traffic units received to generate $N-1$ linearly independent combinations (A process known as network coding [9]). These combinations must also be linearly independent from the original traffic units received from the members. Afterwards, the $N-1$ combinations are groomed and delivered back to the members using light-tree(s). Each of the members will be able to recover the original traffic units by linearly combining its own traffic unit with the received combinations (e.g., solving $N$ linearly independent combinations), see Fig. (1.b). For simplicity, we assume that the linear combinations are performed using coefficients taken from a field of size 2. Also we make the assumption that all the members in a session have the same traffic demand. This assumption is needed to facilitate network coding at the hub node by performing bitwise XOR on equal sized data units.

\section{A Comparative Example}

Consider the example shown in Fig. 1, where nodes $A, B$ and $C$ are members of a many-to-many session. Each one of the members needs to send one unit of traffic denoted as $a, b$ and $c$ respectively to the other two members. For the sake of this example, we assume that the capacity of a wavelength channel (grooming factor) is two units of traffic. In the nonsplitting network case, Figure 1.(a) illustrates the optimal provisioning of the session by the lightpath approach, which requires a total of 6 transceivers. In the splitting network case, Figure 1.(b) illustrates the provisioning of the session by the hub-based approach. Note that each of the members $A$ and $C$ will be able to recover the original traffic units by adding $a+c$ with their own traffic unit modulo 2 . This approach requires a total of 7 transceivers, which costs one more transceiver than the lightpath approach. On the other hand, if $a, b$ and $c$ are two units of traffic instead of one, then the optimal provisioning by the lightpath approach, in the non-splitting network case, is shown in Figure 1.(c), which requires a total of 12 transceivers. However, in the splitting network case, the hub-based approach, as shown in Figure 1.(d), requires a total of 10 transceivers, which saves 2 transceivers compared to the lightpath approach.

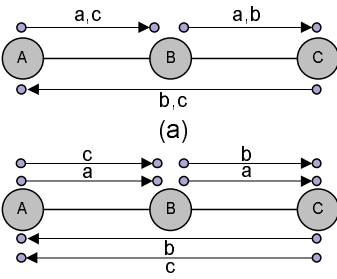

(c)

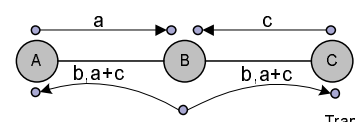

(b)

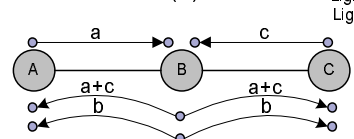

(d)
Fig. 1. Lightpath Approach vs. Hub-based Approach

\section{HUB-BASED APPROACH: MILP FORMULATION}

The many-to-many traffic grooming problem under the hubbased approach is formally defined as follows: Given the network topology, number of wavelengths per fiber, grooming factor and a set of many-to-many session requests with arbitrary sub-wavelength traffic demands, determine, for each session, how to:

1) Optimally select a hub node.

2) Optimally find a many-to-one tree to deliver the traffic from the members to the hub node, we call it membersto-hub journey.

3) Optimally find a multicast tree to deliver the linear combinations from the hub node back to the members, we call it hub-to-members journey.

The members-to-hub journey may traverse multiple lightpaths from each of the members to the hub, while the hub-tomembers journey may traverse multiple light-tree(s) from the hub to the members. We note that a light-tree in our model is associated with a session, e.g, when we say "light-tree belonging to session $s_{a}$ " we mean a light-tree that it is rooted at the hub of $s_{a}$ and its leaves are the members of $s_{a}$. Lightpaths and light-trees may groom traffic from different sessions and traffic from different members within a session. The objective of the optimization procedure is to minimize the total number of transceivers required.

In this section, we formulate an MILP, which we refer to as the HUB-MILP, to solve the many-to-many traffic grooming problem in splitting networks under the hub-based approach. The following notations are used in the MILP:

$N \quad$ total number of nodes in the network.

$P_{m n} \quad$ binary number equals to 1 if there is a directed fiber link from node $m$ to node $n$.

$W \quad$ number of wavelengths per fiber, which we set large enough to guarantee a feasible solution.

$g \quad$ grooming factor. 
$K \quad$ total number of sessions in the network.

$m_{s_{a}} \quad$ the set of members in session $s_{a}$, where $1 \leq a \leq K$.

$N_{s_{a}} \quad$ number of members in session $s_{a} ; N_{s_{a}}=\left|m_{s_{a}}\right|$.

$t_{s_{a}} \quad$ number of units of traffic demanded by each of the members in session $s_{a}$, where $1 \leq t_{s_{a}} \leq g$.

$B_{l}^{s_{a}} \quad$ binary number equals to 1 if session $s_{a}$ has $l$ as one of its members.

$Q \quad$ A very large integer; $Q \geq K \cdot N$.

$T R_{n} \quad$ number of transceivers at node $n$.

$L P_{i j}^{w} \quad$ number of lightpaths from node $i$ to node $j$ on wavelength $w$.

$L P_{i j} \quad$ number of lightpaths from node $i$ to node $j$ on all wavelengths; $L P_{i j}=\sum_{w} L P_{i j}^{w}$.

$F_{m n}^{i j, w} \quad$ binary number equals to 1 if there is a lightpath from $i$ to $j$ that uses fiber $m n$ on wavelength $w$.

$Z_{i j}^{s_{a}, k} \quad$ binary number equals to 1 if the traffic stream originating from member $k \in m_{s_{a}}$ and terminating at the hub of $s_{a}$ is using a lightpath from $i$ to $j$.

$X_{i j}^{s_{a}} \quad$ real number equals to the amount of traffic carried on lightpath(s) from $i$ to $j$ due to all $k \in m_{s_{a}}$.

$I_{h}^{s_{a}} \quad$ binary number equals to 1 if node $h$ is the hub node for session $s_{a}$.

$E_{s_{b}}^{s_{a}, h} \quad$ binary number equals to 1 if sessions $s_{a}$ and $s_{b}$ share node $h$ as their hub node.

$E_{s_{b}}^{s_{a}} \quad$ binary number equals to 1 if sessions $s_{a}$ and $s_{b}$ share the same hub node. We note that this is the disjunction of $E_{s_{b}}^{s_{a}, h}$ for all values of $h ; E_{s_{a}}^{s_{a}}=1$.

$L T_{s_{a}}^{w} \quad$ number of light-trees belonging to session $s_{a}$ on wavelength $w$.

$L T_{s_{a}} \quad$ number of light-trees belonging to session $s_{a}$ on all wavelengths; $L T_{s_{a}}=\sum_{w} L T_{s_{a}}^{w}$.

$A_{h}^{s_{a}} \quad$ real number equals to the product of $L T_{s_{a}}$ and $I_{h}^{s_{a}}$. $R_{m n}^{s_{a}, l, w}$ binary number equals to 1 if there is a light-tree belonging to session $s_{a}$ with root (hub of $s_{a}$ ) to leaf (member $l \in m_{s_{a}}$ ) path that uses fiber $m n$ on wavelength $w$.

$R_{m n}^{s_{a}, w} \quad$ binary number equals to 1 if at least one of the root (hub of $s_{a}$ ) to leaf (member $l \in m_{s_{a}}$ ) paths of a light-tree belonging to session $s_{a}$ uses fiber link $m n$ on wavelength $w$.

$U_{s_{b}}^{s_{a}} \quad$ binary number equals to 1 if session $s_{a}$ is routed on a light-tree belonging to session $s_{b}$.

$T_{s_{b}}^{s_{a}} \quad$ real number equals to the amount of traffic carried on light-tree(s) belonging to session $s_{b}$ due to all members in session $s_{a}$.

\section{Objective Function:}

$$
\text { Minimize: } \sum_{n} T R_{n}
$$

\section{Subject to:}

- Number of transceivers:

The following constraint ensures that at the source and at the destination of each lightpath there is a transceiver present. Also, it ensures that at the root and at the leaves of each light-tree there is a transceiver present.

$$
\begin{aligned}
T R_{i} & \geq \sum_{j: j \neq i}\left(L P_{i j}+L P_{j i}\right)+\sum_{s_{a}} L T_{s_{a}} B_{i}^{s_{a}} \\
& +\sum_{s_{a}: i \notin m_{s_{a}}} A_{i}^{s_{a}} \quad \forall i
\end{aligned}
$$

The nonlinear term $A_{i}^{s_{a}}$ can be computed using the following set of linear constraints (together with the minimization in the objective function):

$$
\begin{gathered}
A_{i}^{s_{a}} \geq Q I_{i}^{s_{a}}-Q+L T_{s_{a}} \quad \forall s_{a}, i \\
A_{i}^{s_{a}} \leq L T_{s_{a}} \quad \forall s_{a}, i
\end{gathered}
$$

- Lightpath level constraints:

$$
\begin{gathered}
\sum_{m: P_{m i}=1} F_{m i}^{i j, w}=\sum_{n: P_{j n}=1} F_{j n}^{i j, w}=0 \quad \forall i, j, w \\
\sum_{n: P_{i n}=1} F_{i n}^{i j, w}=\sum_{m: P_{m j}=1} F_{m j}^{i j, w}=L P_{i j}^{w} \quad \forall i, j, w \\
\sum_{m: P_{m x}=1} F_{m x}^{i j, w}=\sum_{n: P_{x n}=1} F_{x n}^{i j, w} \quad \forall i, j, w, x \neq(i, j)
\end{gathered}
$$

Constraints (4), (5) and (6) together ensure that for each lightpath from $i$ to $j$ there is a corresponding physical path from $i$ to $j$ not using fibers coming into $i$ or outgoing from $j$ and satisfying the wavelength continuity.

\section{- Light-tree level constraints:}

In this set of constraints, we visualize a light-tree belonging to session $s_{a}$ as a set of paths, each originating from the root of the light-tree (hub of $s_{a}$ ) and terminating at one of its leaves (one of the members of $s_{a}$ ). We refer to these paths as root-to-leaf paths. The following constraint ensures that no root-to-leaf path is routed on a fiber link coming into the root.

$$
\sum_{m: p_{m h}=1} R_{m h}^{s_{a}, l, w} \leq\left(1-I_{h}^{s_{a}}\right) Q \quad \forall s_{a}, l \in m_{s_{a}}, h, w
$$

The following constraint ensures that no root-to-leaf path is routed on a fiber link outgoing from the leaf.

$$
\sum_{n: p_{l n}=1} R_{l n}^{s_{a}, l, w}=0 \quad \forall s_{a}, l \in m_{s_{a}}, w
$$

The following constraints ensure that for each leaf of a light-tree there should be a root-to-leaf path originating from the root.

$$
\begin{gathered}
\forall s_{a}, l \in m_{s_{a}}, h \neq l, w: \\
\sum_{n: p_{h n}=1} R_{h n}^{s_{a}, l, w} \geq L T_{s_{a}}^{w}-\left(1-I_{h}^{s_{a}}\right) Q \\
\sum_{n: p_{h n}=1} R_{h n}^{s_{a}, l, w} \leq L T_{s_{a}}^{w}+\left(1-I_{h}^{s_{a}}\right) Q
\end{gathered}
$$

The following constraints ensure that for each leaf of a light-tree there should be a root-to-leaf path terminating at the leaf.

$$
\sum_{m: p_{m l}=1} R_{m l}^{s_{a}, l, w} \geq L T_{s_{a}}^{w}-Q I_{l}^{s_{a}} \quad \forall s_{a}, l \in m_{s_{a}}, w
$$




$$
\sum_{m: p_{m l}=1} R_{m l}^{s_{a}, l, w} \leq L T_{s_{a}}^{w}+Q I_{l}^{s_{a}} \quad \forall s_{a}, l \in m_{s_{a}}, w
$$

The following constraints ensure the wavelength continuity of a root-to-leaf path.

$$
\begin{gathered}
\forall s_{a}, l \in m_{s_{a}}, w, x \neq l: \\
\sum_{m: p_{m x}=1} R_{m x}^{s_{a}, l, w} \leq \sum_{n: p_{x n}=1} R_{x n}^{s_{a}, l, w} \\
\sum_{m: p_{m x}=1} R_{m x}^{s_{a}, l, w} \geq \sum_{n: p_{x n}=1} R_{x n}^{s_{a}, l, w}-Q I_{x}^{s_{a}}
\end{gathered}
$$

The following constraints ensure that the same wavelength is used on all root-to-leaf paths that belong to the same light-tree.

$$
\begin{gathered}
\forall s_{a},(l, k) \in m_{s_{a}}, w: \\
\sum_{m: p_{m l}=1} R_{m l}^{s_{a}, l, w} \geq \sum_{m: p_{m k}=1} R_{m k}^{s_{a}, k, w}-\left(I_{l}^{s_{a}}+I_{k}^{s_{a}}\right) Q \\
\sum_{m: p_{m l}=1} R_{m l}^{s_{a}, l, w} \leq \sum_{m: p_{m k}=1} R_{m k}^{s_{a}, k, w}+\left(I_{l}^{s_{a}}+I_{k}^{s_{a}}\right) Q
\end{gathered}
$$

The following constraints set the variable $R_{m n}^{s_{a}, w}$ as the disjunction of $R_{m n}^{s_{a}, l, w}$ for all values of $l$.

$$
\begin{gathered}
R_{m n}^{s_{a}, w} \geq \sum_{l \in m_{s_{a}}} R_{m n}^{s_{a}, l, w} / Q \quad \forall s_{a}, w, m, n: p_{m n}=1 \\
R_{m n}^{s_{a}, w} \leq \sum_{l \in m_{s_{a}}} R_{m n}^{s_{a}, l, w} \quad \forall s_{a}, w, m, n: p_{m n}=1
\end{gathered}
$$

The following constraint ensures that for any wavelength $w$ on any fiber link $m n$ no more than one lightpath or light-tree can be present.

$$
\sum_{s_{a}} R_{m n}^{s_{a}, w}+\sum_{i} \sum_{j} F_{m n}^{i j, w} \leq 1 \quad \forall w, m, n: p_{m n}=1
$$

\section{- Hub node selection constraints:}

The following constraint ensures that there is exactly one hub node for each session.

$$
\sum_{h} I_{h}^{s_{a}}=1 \quad \forall s_{a}
$$

The following constraints set the variable $E_{s_{b}}^{s_{a}, h}$ as the conjunction of the variables $I_{h}^{s_{a}}$ and $I_{h}^{s_{b}}$.

$$
\begin{aligned}
& E_{s_{b}}^{s_{a}, h} \leq\left(I_{h}^{s_{a}}+I_{h}^{s_{b}}\right) / 2 \quad \forall s_{a}, s_{b}, h \\
& E_{s_{b}}^{s_{a}, h} \geq I_{h}^{s_{a}}+I_{h}^{s_{b}}-1 \quad \forall s_{a}, s_{b}, h
\end{aligned}
$$

The following constraints set the variable $E_{s_{b}}^{s_{a}}$ as the disjunction of $E_{s_{b}}^{s_{a}, h}$ for all values of $h$.

$$
\begin{gathered}
E_{s_{b}}^{s_{a}} \geq \sum_{h} E_{s_{b}}^{s_{a}, h} / Q \quad \forall s_{a}, s_{b} \\
E_{s_{b}}^{s_{a}} \leq \sum_{h} E_{s_{b}}^{s_{a}, h} \quad \forall s_{a}, s_{b}
\end{gathered}
$$

\section{- Members-to-hub journey constraints:}

In this set of constraints, we visualize the membersto-hub journey of a session as a set of streams, each originating from a member and terminating at the hub.
Each of these streams, which we refer to as member-tohub streams, may traverse multiple lightpaths from the member to the hub. The following constraint ensures that a member-to-hub stream cannot be routed on a lightpath coming into the member.

$$
\sum_{i: i \neq k} Z_{i k}^{s_{a}, k}=0 \quad \forall s_{a}, k \in m_{s_{a}}
$$

The following constraint ensures that a member-to-hub stream cannot be routed on a lightpath outgoing from the hub.

$$
\sum_{i: i \neq h} Z_{h i}^{s_{a}, k} \leq\left(1-I_{h}^{s_{a}}\right) Q \quad \forall s_{a}, k \in m_{s_{a}}, h \neq k
$$

The following constraint ensures that for each memberto-hub stream, there is a lightpath originating from the member unless it is the hub.

$$
\sum_{i: i \neq k} Z_{k i}^{s_{a}, k}=1-I_{k}^{s_{a}} \quad \forall s_{a}, k \in m_{s_{a}}
$$

The following constraint ensures that for each memberto-hub stream, there is a lightpath terminating at the hub.

$$
\sum_{i: i \neq h} Z_{i h}^{s_{a}, k} \geq I_{h}^{s_{a}} \quad \forall s_{a}, k \in m_{s_{a}}, h \neq k
$$

The following constraint ensures the continuity of a member-to-hub stream on multiple lightpaths.

$$
\sum_{i: i \neq x} Z_{i x}^{s_{a}, k}=\sum_{j: j \neq(x, k)} Z_{x j}^{s_{a}, k}+I_{x}^{s_{a}} \quad \forall s_{a}, k \in m_{s_{a}}, x \neq k
$$

The following constraint determines the exact amount of traffic carried on lightpath(s) from node $i$ to node $j$ due to all members $k \in m_{s_{a}}$.

$$
X_{i j}^{s_{a}}=t_{s_{a}} \times \sum_{k \in m_{s_{a}}} Z_{i j}^{s_{a}, k} \quad \forall s_{a}, i, j
$$

The following constraint determines the total number of lightpath(s) needed from node $i$ to node $j$.

$$
L P_{i j} \geq\left(\sum_{s_{a}} X_{i j}^{s_{a}}\right) / g \quad \forall i, j
$$

\section{- Hub-to-members journey constraints:}

In this set of constraints, we determine which light-tree(s) are used in the hub-to-members journey of a session. The following constraint ensures that the hub-to-members journey of a session cannot be routed on a light-tree of another session unless the two sessions share the same hub node.

$$
U_{s_{b}}^{s_{a}} \leq E_{s_{b}}^{s_{a}} \quad \forall s_{a}, s_{b}
$$

The following constraint ensures that each member of a session must be reached by at least one of the light-trees used in the hub-to-members journey of the session.

$$
\sum_{s_{b}: l \in m_{s_{b}}} U_{s_{b}}^{s_{a}} \geq 1 \quad \forall s_{a}, l \in m_{s_{a}}
$$


The following constraint determines the exact amount of traffic carried on light-tree(s) belonging to session $s_{b}$ due to all members in session $s_{a}$.

$$
T_{s_{b}}^{s_{a}}=U_{s_{b}}^{s_{a}} \times t_{s_{a}} \times\left(N_{s_{a}}-1\right) \quad \forall s_{a}, s_{b}
$$

This represents the total amount of traffic after coding the traffic units transmitted by the members of session $s_{a}$ at the hub node of session $s_{b}$. The following constraint determines the total number of light-trees needed for session $s_{a}$.

$$
L T_{s_{a}} \geq\left(\sum_{s_{b}} T_{s_{a}}^{s_{b}}\right) / g \quad \forall s_{a}
$$

\section{Numerical Results}

In this section, we compare the lightpath approach and the hub-based approach using LP-MILP and HUB-MILP, respectively. In our experiments, we consider the network shown in Fig. 2 which is based on the Abilene Research Network [11]. Each link is composed of two unidirectional fibers in opposite directions, and the number of wavelength channels in each fiber, $W$, is set to 4 . Traffic demands are integer multiples of OC-3 rates, while a wavelength channel has a capacity of OC-48; hence, the grooming factor, $g$, is 16 .

We run 6 random experiments as follows, each experiment has 5 many-to-many sessions where the size of each session is randomly selected between $[2,4]$. The members in each session are randomly selected between $[0,9]$ and the traffic demanded by each of the members in a session is randomly selected between $[1,16]$. For each experiment, we define $t=\sum_{s_{a}} N_{s_{a}} * t_{s_{a}} / \sum_{s_{a}} N_{s_{a}}$, to be the average amount of traffic demanded by a member in this experiment. The optimal solution from LP-MILP and HUB-MILP for each of these experiments is obtained using the CPLEX solver [12]. The number of transceivers $(T R)$ required by each of the experiments is shown in Table I. Due to space limitations, we do not show the actual provisioning of sessions in the 6 experiments. However, Fig. 2 illustrates the provisioning of sessions 1 and 4 in Exp. \#1 (shown in Table II) using the HUB-MILP.

From the results we conclude that the lightpath approach is a better choice when traffic demands of sessions are relatively low $(t<g / 2)$, see Exps. 5 and 6 , while the hub-based approach is a better choice when traffic demands of sessions are relatively high $(t>g / 2)$, see Exps. 2 and 3. The reason for this is that light-trees are not efficient in grooming traffic and therefore when traffic demands are relatively low $(t<g / 2)$, the hub-based approach do not perform well. On the other hand, lightpaths are very efficient in grooming traffic and therefore the lightpath approach performs better when traffic demands are relatively low $(t<g / 2)$.

\section{CONCLUSIONS}

In this paper, we have studied the many-to-many traffic grooming problem in both splitting and non-splitting WDM networks. In non-splitting networks, we have generalized the model in [8] to provide an optimal lightpath approach. In splitting networks, we have introduced the hub-based approach that combines optical splitting and network coding to provision the sessions. We have concluded that the lightpath approach is a better choice when traffic demands of sessions are relatively low $(t<g / 2)$ while the hub-based approach is a better choice when traffic demands of sessions are relatively high $(t>g / 2)$.

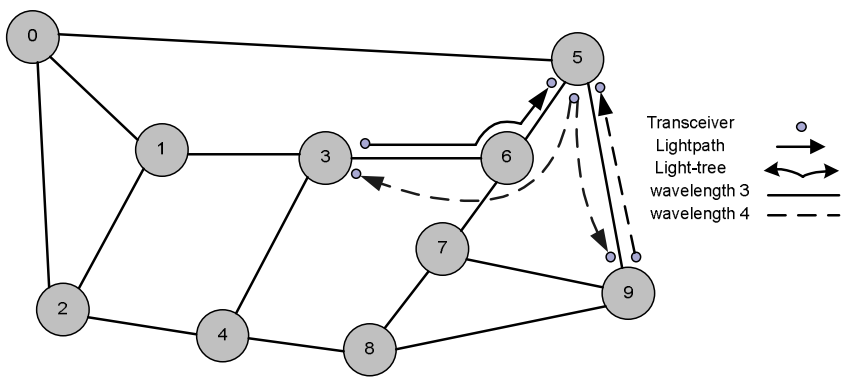

Fig. 2. Abilene Research Network (with two additional links): Provisioning of $s_{1}$ and $s_{4}$ in Exp. \#1 using the HUB-MILP. Note that the two sessions are groomed on lightpath $3 \rightarrow 5$ and on light-tree $5 \rightarrow(3,9)$ where $\operatorname{hub}\left(s_{1}\right)=\operatorname{hub}\left(s_{4}\right)=5$

TABLE I

NUMBER OF TRANSCEIVERS (TR) COMPARISON BETWEEN THE LIGHTPATH AND THE HUB-BASED APPROACHES

\begin{tabular}{c|c|c|c|c|c|c}
\hline Exp \# & 1 & 2 & 3 & 4 & 5 & 6 \\
\hline$t$ & 8.3 & 11.8 & 9.8 & 7.5 & 5.3 & 4.4 \\
\hline LP-MILP(TR) & 28 & 36 & 38 & 30 & 20 & 18 \\
\hline HUB-MILP(TR) & 28 & 32 & 34 & 29 & 23 & 22 \\
\hline
\end{tabular}

TABLE II

INPUT PARAMETERS FOR EXPERIMENT \#1

\begin{tabular}{|c|c|c|}
\hline \hline Session & Members & Traffic Demands \\
\hline$s_{1}$ & $(3,5)$ & 3 \\
\hline$s_{2}$ & $(1,2,7)$ & 14 \\
\hline$s_{3}$ & $(0,6,8)$ & 7 \\
\hline$s_{4}$ & $(3,5,9)$ & 5 \\
\hline$s_{5}$ & $(4,9)$ & 12 \\
\hline
\end{tabular}

\section{REFERENCES}

[1] E. Modiano, "Traffic Grooming in WDM Networks" IEEE Communications pp. 124-129, July 2001.

[2] O. Gerstel, R. Ramaswami, and G. Sasaki, "Cost-effective Traffic Grooming in WDM Rings," IEEE/ACM Transactions on Networking, Oct. 2000.

[3] K. Zhu and B. Mukherjee,"Traffic Grooming in a WDM Mesh Network," IEEE Journal on Selected Areas in Communications, Jan 2002.

[4] R. Dutta and G. N. Rouskas, "Traffic grooming in WDM networks: past and future," IEEE Network, vol. 16, no. 6, pp. 4656, Nov./Dec. 2002.

[5] R. Ul-Mustafa and A. E. Kamal,"Design and provisioning of WDM networks with multicast traffic grooming," IEEE J. Select. Areas Commun., vol. 24, no. 4, pp. 3753, Apr. 2006.

[6] G. Chowdhary and C. S. R. Murthy,"Grooming of multicast sessions in wdm mesh networks," in Workshop on Traffic Grooming, 2004.

[7] Kamal, A. E., "Algorithms for Multicast Traffic Grooming in WDM Mesh Networks," IEEE Communications Nov. 2006.

[8] R. Ul-Mustafa and A. E. Kamal,"Many-to-one Traffic Grooming with Aggregation in WDM Networks "IEEE Journal on Selected Areas in Communication, vol. 24, no. 8, August 2006.

[9] R. Ahlswede, N. Cai, S. R. Li, and R. W. Yeung,"Network information flow" IEEE Trans. Inf. Theory vol. 46, no. 4, pp. 12041216, Jul. 2000.

[10] http://ecpe.ece.iastate.edu/lan/downloads/LP-MILP.pdf

[11] The Abilene Research Network, http://abilene.internet2.edu/.

[12] http://www.ilog.com/products/cplex/ 